Wilfrid Laurier University

Scholars Commons @ Laurier

Physics and Computer Science Faculty

Publications

Physics and Computer Science

$1-6-2020$

Dynamics and Stability of the Two Body Problem with Yukawa Correction

Eli Cavan

loannis Haranas

loannis Gkigkitzis

Follow this and additional works at: https://scholars.wlu.ca/phys_faculty

Part of the Computer Sciences Commons, and the Physics Commons 


\title{
Dynamics and Stability of the Two Body Problem with Yukawa Correction
}

\author{
Eli Cavan* and Ioannis Haranas ${ }^{\dagger}$ \\ Wilfrid Laurier University Department of Physics \& Computer Science \\ Waterloo, ON, CANADA \\ Ioannis Gkigkitzis \\ NOVA, Department of Mathematics, \\ 8333 Little River Turnpike, \\ Annandale, VA 22003, USA
}

(Dated: January 6, 2020)

\begin{abstract}
We explore the dynamics and stability of the two body problem by modifying the Newtonian potential with the Yukawa potential. This model may be considered a theory of modified gravity; where the interaction is not simply the kepler solution for large distance. The stability is investigated by deriving the Jacobian of the linearized matrix equation and evaluating the eigenvalues of the various equilibrium points calculated during the analysis. The subcases of a purely Yukawa and purely Newtonian potential are also explored.
\end{abstract}

Key Words: Gravity — Two body Problem — Stability - Yukawa Potential

\section{INTRODUCTION}

Many of today's theories predict corrections to the theory of Gravitation. The Yukawa Potential has been studied by a number of researchers as a model to describe deviations from Newton's inverse square law. Theories like Scalar-Tensor-Vector Gravity Theory (Moffat, 2006) predict a Yukawa-like fifth force. In (Iorio 2007) the author finds constraints on the range of the Yukawa interaction $\lambda$ by comparing corrections to the Newtonian-Einsteinian secular rates of the perihelia of Mercury. (Brownstein \& Moffat, 2006) studied the Yukawa potential as a modification to Newton's constant $G$, introducing a distance varying gravitational acceleration corrected by the Yukawa force. In (De Laurentis et al. 2018) the authors solve the geodesic equation of a particle subjected to a Yukawa corrected gravitational field.

In this contribution we explore the effect of a Yukawa correction to the gravitational force over large distances (binary star-like orbits). In particular, we are able to study the stability of the closed orbit solutions, and compare them to the classical kepler problem. In particular, we are able to prove using Bertrand's theorem that closed orbits exists for appropriate values of $r$. This models is interesting as the simplest correction to gravity over large distance that one can imagine; future astrophysical experiments will ultimately dictate the validity of the model.

This paper specifically builds on work from (Haranas et al 2011), (Haranas et al 2016) (Haranas \& Ragos 2011) in which the authors calculate various celestial orbital properties under the correction of a Yukawa term. In particular in (Haranas et al 2011) the authors compute the correction to the anomalistic period, in (Haranas et

\footnotetext{
* cava0920@mylaurier.ca

$\dagger$ iharanas@wlu.ca

¥ igkigkitzis@nvcc.edu
}

al 2016) the authors calculate the corrected mean motion and in (Haranas \& Ragos 2011) the authors study the effects of the Yukawa correction on the orbits of satellites. ( E.G. Adelberger et al 2009) and (Borka et al 2013) were also important papers for confining the range (or coupling) of the Yukawa force for short and large range distances respectively. (Borka et al 2013) is of particular importance as the authors study star-like orbits, and show through numerical simulations that closed orbits are a possible solution for a mass in a Yukawa corrected gravitational potential.

The Yukawa force is particularly valid as a long range force, as early estimates of its range $\lambda$ from (Brownstein \& Moffat 2006) and (Haranas \& Ragos 2011) indicate that $\lambda \geq 10^{15} \mathrm{~m} . \quad \lambda$ is the Compton wavelength $\lambda=\frac{h}{m c}$ of the particle mediating the interaction, for gravity that is the graviton. The Yukawa potential could be used, for example, to model the interaction between massive stars; which are separated on average by a distance of $10^{12} \mathrm{~m}$. Thus, we are interested in studying orbits of size relative to the size of the Solar System; one example we study is the orbit of two stars of mass equal to that of the Sun. The corresponding Newtonian potential is given by

$$
V_{N}(r)=-\frac{G m_{1} m_{2}}{r}
$$

where $r=\left(r_{x}, r_{y}\right)$ and $G=6.6710^{-11} \frac{\mathrm{N} \cdot \mathrm{m}^{2}}{\mathrm{~kg}^{2}}$ is Newton's constant. The form of the potential studied in this report is

$$
V(r)=-\frac{G m_{1} m_{2}}{r}\left(1+\alpha e^{-\frac{r}{\lambda}}\right)=V_{N}(r)+V_{Y k}(r)
$$

Where $V_{Y k}(r)$ is the Yukawa correction to the Newtonian potential, $\alpha=\frac{k_{g} k_{Y k}}{G M m}$ is the coupling constant of the Yukawa force to the Gravitational force (Haranas \& Gkigkitzis 2011) and $\lambda$ is the range of the Yukawa force as previously mentioned. The results from (Brownstein \& Moffat, 2006) and (Haranas \& Ragos, 2011) indicate 
that $\alpha=10^{-8}$ for Solar System orbits. In this contribution, we study the Yukawa correction to the Newtonian gravitational force; we also study the subcases of a purely Yukawa potential and a purely Newtonian potential $\alpha \rightarrow 0$. The dynamics of the two masses is obtained using the Hamiltonian Formulation of Classical Mechanics; a review is found in (Jose \& Saletan, 1998). The two body problem is studied extensively in (Pollard 1966).

\section{HAMILTONIAN FORMULATION}

We can assume the form of the Hamiltonian $H=T+V$ where $T$ is the kinetic energy of both masses and $V$ is the Gravitational potential energy.

$H=\frac{p_{1}^{2}}{2 m_{1}}+\frac{p_{2}^{2}}{2 m_{2}}-\frac{k}{\left|\boldsymbol{r}_{\mathbf{2}}-\boldsymbol{r}_{\mathbf{1}}\right|^{2}}\left(1+\alpha e^{-\frac{\left|\boldsymbol{r}_{\mathbf{2}}-\boldsymbol{r}_{\mathbf{1}}\right|}{\lambda}}\right)\left(\boldsymbol{r}_{\mathbf{2}}-\boldsymbol{r}_{\mathbf{1}}\right)$

Where $p_{i}=m_{i} v_{i}$ is the momentum of each mass and $k=$ $G m_{1} m_{2}$. Note that $\boldsymbol{p}_{\boldsymbol{i}}=\left(p_{i x}, p_{i y}\right)$ and $\boldsymbol{r}_{\boldsymbol{i}}=\left(r_{i x}, r_{i y}\right)$; the bold font indicates a vector. Changing to the centre of mass frame gives

$$
H=\frac{p^{2}}{2 \mu}-\frac{k}{r}\left(1+\alpha e^{-\frac{r}{\lambda}}\right)
$$

Here we have defined $\mu=\frac{m_{1} m_{2}}{m_{1}+m_{2}}$ as the reduced mass of the system and $r=|\boldsymbol{r}|$. Next we must switch to polar coordinates; this procedure yields

$$
H=\frac{1}{2 \mu}\left(p_{r}^{2}+\frac{p_{\theta}^{2}}{r^{2}}\right)-\frac{k}{r}\left(1+\alpha e^{-\frac{r}{\lambda}}\right)
$$

Following (Goldstein, 1980) given that the Hamiltonian is cyclic is $\theta$ (i.e the Hamiltonian does not depend explicitly on $\theta$ ) we can write Hamilton's equations for $\theta$ as:

$$
\begin{gathered}
\dot{\theta}=\frac{\partial H}{\partial p_{\theta}}=\frac{p_{\theta}}{\mu r^{2}} \\
\dot{p_{\theta}}=-\frac{\partial H}{\partial \theta}=0
\end{gathered}
$$

And so $p_{\theta}=l$ is constant. Given that Hamilton's equations have this form we can write our Hamiltonian as

$$
H=\frac{1}{2 \mu}\left(p_{r}^{2}+\frac{l^{2}}{r^{2}}\right)-\frac{k}{r}\left(1+\alpha e^{-\frac{r}{\lambda}}\right)
$$

Where $l$ is the angular momentum of the binary system. Hamilton's equations for $r$ become

$$
\begin{aligned}
\dot{r} & =\frac{\partial H}{\partial p_{r}}=\frac{p_{r}}{\mu} \\
\dot{p_{r}} & =-\frac{\partial H}{\partial r}=\frac{l^{2}}{\mu r^{3}}-\frac{k}{r^{2}}\left(1+\alpha\left(1+\frac{r}{\lambda}\right) e^{-\frac{r}{\lambda}}\right)
\end{aligned}
$$

It can be shown that $H(t)=H\left(t_{0}\right)=h$ is constant during the motion of the masses; see (Pollard,1966) for example.

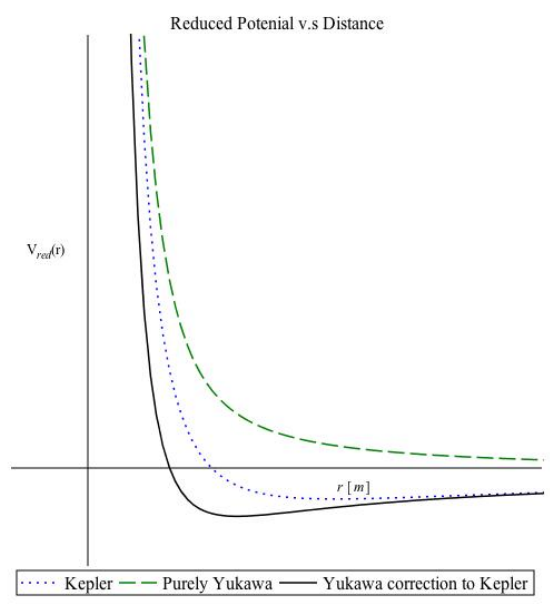

FIG. 1. The Reduced Potential Given Fixed Initial Energy

Since $p_{r}^{2} \geq 0$ we have that the total energy of the system is bounded by

$$
h \geq \frac{l^{2}}{2 \mu r^{2}}-\frac{k}{r}\left(1+\alpha e^{-\frac{r}{\lambda}}\right)
$$

Here we have defined the reduced potential which is common to the Kepler problem (with the Yukawa correction).

$$
V_{r}(r)=\frac{l^{2}}{2 \mu r^{2}}-\frac{k}{r}\left(1+\alpha e^{-\frac{r}{\lambda}}\right)
$$

We can graph the function for fixed $h$ giving us the permissible regions of motion. The graph is shown in figure 1 . Note that $\mu>0, \lambda>0$ and $\alpha>0$.

In figure 1 we have graphed the reduced potential for a given fixed initial energy $h$ measured in joules $(\mathrm{J})$, i.e equation (11). The dotted line refers to the kepler problem, the dashed line is a purely Yukawa potential and the solid line refers to the Newtonian plus Yukawa correction. Points that lie above the graphs represent possible unbounded motion for a massive body with given initial energy and distance from the central body. For small $r$ it is clear that bounded motion can exist.

\section{THE LINEARIZATION MATRIX}

Following (Meiss 2007), to determine the stability of the equilibrium points of the system, we must form a matrix differential equation using the equations of motion of the system (Hamilton's equations for $r$ ); given by (9)(10). The linear system has the form

$$
\frac{d}{d t}\left(\begin{array}{c}
r \\
p_{r}
\end{array}\right)=\left[\begin{array}{ll}
\frac{\partial f}{\partial r} & \frac{\partial f}{\partial p_{r}} \\
\frac{\partial g}{\partial r} & \frac{\partial g}{\partial p_{r}}
\end{array}\right]\left(\begin{array}{c}
r \\
p_{r}
\end{array}\right)
$$

Where $f\left(r, p_{r}\right)=\frac{p_{r}}{\mu}$ and $g\left(r, p_{r}\right)=\frac{l^{2}}{\mu r^{3}}-$ $\frac{k}{r^{2}}\left(1+\alpha\left(1+\frac{r}{\lambda}\right) e^{-\frac{r}{\lambda}}\right)$; i.e the RHS of (9)-(10). Given 
that $\lambda=10^{15} \mathrm{~m}$ for orbits of size comparable to solar system dimensions (Brownstein \& Moffat, 2006), (Haranas \& Ragos, 2011). We assume $\frac{r}{\lambda}$ is a small number we can taylor expand and ignore terms of $O\left(\frac{r^{2}}{\lambda^{2}}\right)$. The expansion about the point $\frac{r}{\lambda}=0$ gives

$$
e^{-\frac{r}{\lambda}} \approx 1-\frac{r}{\lambda}+O\left(\frac{r^{2}}{\lambda^{2}}\right)
$$

And thus $g\left(r, p_{r}\right)=\frac{l^{2}}{\mu r^{3}}-\frac{k}{r^{2}}\left(1+\alpha\left(1+\frac{r}{\lambda}\right)\left(1-\frac{r}{\lambda}\right)\right)$ and so the Jacobian matrix takes the form:

$$
\left(\begin{array}{c}
\dot{r} \\
\dot{p_{r}}
\end{array}\right)=\left[\begin{array}{cc}
0 & \frac{1}{\mu} \\
-\frac{3}{\mu r^{4}}+\frac{2 k}{r^{3}}(1+\alpha) & 0
\end{array}\right]\left(\begin{array}{c}
r \\
p_{r}
\end{array}\right)
$$

Where again, we have ignored terms $O\left(\frac{r^{2}}{\lambda^{2}}\right)$. The equilibrium points are points $(a, b)$ such that $f(a, b)=0$ and $g(a, b)=0$. Thus we can choose $b=p_{r}$ as one coordinate of our equilibrium point. For the value of the equilibrium $r$ coordinate (i.e $r_{e q}=a$ ) using (10) we have

$$
\begin{aligned}
\frac{l^{2}}{\mu r^{3}}-\frac{k}{r^{2}}\left(1+\alpha\left(1+\frac{r}{\lambda}\right)\left(1-\frac{r}{\lambda}\right)\right) & =0 \\
\frac{l^{2}}{\mu r^{3}}-\frac{k}{r^{2}}\left[1+\alpha\left(1-\frac{r^{2}}{\lambda^{2}}\right)\right] & =0
\end{aligned}
$$

And so ignoring higher order terms the equilibrium points are determined by solving

$$
\frac{l^{2}}{\mu}-k r(1+\alpha)=0
$$

Solving this equation gives us equilibrium solution:

$$
r_{e q}=a=\frac{l^{2}}{\mu k(1+\alpha)}
$$

We can now test for stability by choosing values of $\mu$, $\alpha, k, l$ and $\lambda$ and finding the eigenvalues of the Jacobian matrix (15) after substituting the equilibrium solution found above. Recall that the eigenvalues $\beta_{1}, \beta_{2}$ are found by solving the equation

$$
\operatorname{det}\left|J-\beta I_{2 x 2}\right|=0
$$

Here $I_{2 x 2}$ refers to the $2 x 2$ identity matrix. The characteristic equation (the eigenvalue equation) becomes

$$
\beta^{2}-\frac{\mu^{2} k^{4}(1+\alpha)^{4}}{l^{6}}=0
$$

The stability is given by the sign of the eigenvalues (Meiss 2007). For example, if $\beta_{1}>\beta_{2}>0$, than the equilibrium is unstable. If $\beta_{1}<\beta_{2}<0$ then the equilibrium is stable. Imaginary eigenvalues are also stable if for $\beta_{1,2}=a \pm i b$ we have that $a<0$ (otherwise it is unstable). In the case when $a=0$; the equilibrium is called a centre (and is stable) (Meiss 2007). Stability refers to how the solution behaves near the equilibrium point; unstable solutions grow to infinity, stable solutions tend to zero and the imaginary cases are the ones which give bound, orbital solutions (specifically the centre case, whereas the stable and unstable imaginary cases are bound solutions tending towards or away from zero).

\section{STABILITY \& BERTRAND'S THEOREM}

We first note that we can study the case for a purely Newtonian Potential by letting $\alpha \rightarrow 0$. Similarly we can study the purely Yukawa potential by ignoring the term derived from the Newtonian potential; the characteristic equations become

$$
\begin{aligned}
& \beta^{2}-\frac{\mu^{2} k^{4}}{l^{6}}=0 \\
& \beta^{2}-\frac{\mu^{2} k^{4} \alpha^{4}}{l^{6}}=0
\end{aligned}
$$

These expressions take into account the fact that our new equilibrium points are

$$
\begin{aligned}
& a_{N}=\frac{l^{2}}{\mu k} \\
& a_{Y K}=\frac{l^{2}}{\mu k \alpha}
\end{aligned}
$$

Where $\left(a, p_{r}\right)$ are the equilibrium points of the systems studied.

We now proceed to evaluate (19), (20) and (21) with the equilibrium solutions to determine the stability of the equilibrium points. Evaluating (19) gives us eigenvalues:

$$
\beta= \pm i \frac{(1+\alpha)^{2} k^{2} \mu^{2}}{l^{3}}
$$

Similarly for the Newtonian and purely Yukawa cases:

$$
\begin{aligned}
& \beta_{N}= \pm i \frac{k^{2} \mu^{2}}{l^{3}} \\
& \beta_{Y K}= \pm i \frac{\alpha^{2} k^{2} \mu^{2}}{l^{3}}
\end{aligned}
$$

And thus the equilibrium points for the purely Yukawa, Newtonian and the Newton + Yukawa Potential remain centre solutions. This would imply that motion is restricted to ellipses about the equilibrium point; and so we could have orbits near the equilibrium point (further away from the equilibrium point we would have unbounded solutions, which can we seen in figure 1). This proves that for small $r$ we have stable, closed orbits. However, Bertrand's theorem says the only potentials which allow closed and bounded solutions are the Newtonian and Harmonic oscillator potentials. Thus, we confirm that for small $r$ our equations obey the conditions of this theorem. We also derive the orbit 
equation for each case.

For the orbit equation, following (Goldstein 1980) the orbit equation for a keplerian problem can be written as

$$
\frac{d^{2} u}{d \theta^{2}}+u=-\frac{\mu}{l^{2}} \frac{d}{d u} V\left(\frac{1}{u}\right)
$$

where $r=\frac{1}{u}$. This can be derived using Hamiltonian's equations and the fact that

$$
\frac{d r}{d t}=\frac{d r}{d \theta} \frac{d \theta}{d t}=\frac{d r}{d \theta} \dot{\theta}
$$

where $\dot{\theta}=\frac{l}{\mu r^{2}}$. After subsequent differentiation by $r$, you arrive at the orbit equation. For our modified potential we have

$$
V\left(\frac{1}{u}\right)=-k u\left(1+\alpha e^{-\frac{1}{\lambda u}}\right)
$$

for small $\frac{r}{\lambda}$ (i.e using the approximation (14)):

$$
\begin{aligned}
\frac{d^{2} u}{d \theta^{2}}+u & =-\frac{\mu}{l^{2}} \frac{d}{d u}\left[-k u-k \alpha u e^{-\frac{1}{\lambda u}}\right] \\
& =-\frac{\mu}{l^{2}}\left[-k-k \alpha\left(1+\frac{1}{\lambda u}\right) e^{-\frac{1}{\lambda u}}\right]
\end{aligned}
$$

Using the approximation in equation (14) and ignoring higher order $O\left(\lambda^{2} u^{2}\right)$ terms we have the orbit equation

$$
\frac{d^{2} u}{d \theta^{2}}+u=\frac{k \mu}{l^{2}}(\alpha+1)
$$

Which has solution

$$
u=\frac{1}{r}=\frac{k \mu}{l^{2}}(\alpha+1)\left[1+e \cos \left(\theta-\theta_{0}\right)\right]
$$

where $e$ is the eccentricity of the orbit. The Newtonian and purely Yukawa cases follow respectively

$$
\begin{aligned}
& u=\frac{1}{r}=\frac{k \mu}{l^{2}}\left[1+e \cos \left(\theta-\theta_{0}\right)\right] \\
& u=\frac{1}{r}=\frac{k \mu \alpha}{l^{2}}\left[1+e \cos \left(\theta-\theta_{0}\right)\right]
\end{aligned}
$$

Finally, to satisfy Bertrand's theorem we must satisfy the condition

$$
\left.\frac{d^{2} V_{r e d}(r)}{d r^{2}}\right|_{r=r_{0}}>0
$$

where the reduced potential is given by (12). After using the approximation for small $\frac{r}{\lambda}$ (i.e (14) and ignoring $\left.O\left(\frac{r^{2}}{\lambda^{2}}\right)\right)$ this condition becomes

$$
\frac{\mu^{3} k^{4}(1+\alpha)^{4}}{l^{6}}>0
$$

which is clearing true since $\mu, \alpha, l, k>0$. Similarly it can be shown that this is true for the Newtonian ( $\operatorname{set} \alpha=0$ ) and purely Yukawa cases. This shows that the Yukawa + Newtonian potential satisfies Bertrand's theorem for small $\frac{r}{\lambda}$. A consequence of Bertrand's theorem is that the ratio $\frac{\omega}{\dot{\theta}}$ is a rational number; where $\dot{\theta}=\frac{l}{\mu r^{2}}$ and $\omega=\frac{2 \pi}{T}$. In a paper to follow will show that $\frac{\omega}{\dot{\theta}}=1-\frac{1}{2} \frac{\lambda^{2}}{r^{2}}$ but since for the solar system theory predicts that $\lambda>>r_{e q}=a$ and therefore for any practical purpose $\frac{\omega}{\dot{\theta}}=1$; this number being rational implies that orbits are bounded. This result is physically reasonable even given our approximations, as mentioned earlier $\lambda \geq 10^{15} \mathrm{~m}$ for Solar System orbits. So for bound orbits near the equilibrium point $r_{e q}=\frac{l^{2}}{\mu k(1+\alpha)}$, we expect $\frac{r}{\lambda}$ to be small. For example, to explore the dynamics of two sun-like stars orbiting at a radius similar to the size of the Solar System; we let $m_{1}=m_{2}=M_{\text {sun }}=10^{30} \mathrm{~kg}$. This would imply $\alpha=10^{-8}, \lambda=10^{15} \mathrm{~m}, k=G m_{1} m_{2}=10^{49} \mathrm{~kg} m^{3} / \mathrm{s}^{2}$, $\mu=\frac{10^{30}}{2} \mathrm{~kg}$ and $l=m r v \approx 10^{45} \mathrm{~kg} \mathrm{~m} / \mathrm{s}$. And so $r_{e q}=2 \cdot 10^{11} \mathrm{~m} \approx 1.3 \mathrm{Au}$; this is comparable to the orbit of Mars, and thus $\frac{r}{\lambda}<<1$ and so the approximations we have been using during this contribution are valid.

\section{CONCLUSION}

We have demonstrated the dynamics and stability of the two body problem with the Yukawa correction to the Newtonian potential. To calculate the former we treated the problem as a modified Kepler problem and derived the equations of motion and reduced potential of the system; which led us to the discussion of unbounded or bounded motion. To demonstrate the latter, stability, we constructed the Linearization matrix and tested the stability of the equilibrium points of the system for a Yukawa correction. We find that the stability of the equilibrium point is a centre solution; which implies stable solutions near the equilibrium point. We repeated the analysis for a purely Yukawa force and find similar results. We also confirm that our modified potential obeys Bertrand's theorem. 
- E.G. Adelberger, J.H. Gundlach, B.R. Heckel, S. Hoedl, S. Schlamminger, Torsion balance experiments: A low-energy frontier of particle physics, Prog. Part. Nucl. Phys. 62 (2009) 102.

- D. Borka, P. Jovanovi?, V. Borka Jovanovi? And A. F. ZAKHAROV, Constraining the range of Yukawa gravity interaction from S2 star orbits, J. Cosmol. Astropart. P. 11 (2013) 050.

- J.B Brownstein \& J.W Moffat, A Gravitational Solution to the Pioneer 10/11 anomaly, Class Quantum Grav., 23 (2006) 3427-3436.

- Goldstein, Herbert, Classical Mechanics, AddisonWesley (1980).

- Haranas I., AND O. Ragos, Yukawa-type effects in satellite dynamics, Astrophys Space Sci (2011) 331: 115119.

- Ioannis Haranas, Omiros Ragos, Vasile Mioc, Yukawa-type potential effects in the anomalistic period of celestial bodies, Astrophys Space Sci (2011) 332: 107-113.

- Ioannis Haranas, Ilias Kotsireas, Guillem Gmez, Mrius J. Fullana, IoAnnis Gkigkitzis, Yukawa effects on the mean motion of an orbiting body, Astrophys Space
Sci (2016) 361:365.

- L. IORIO , Constraints on the range lambda of Yukawalike modifications to the Newtonian inverse-square law of gravitation from Solar System planetary motions, Journal of High Energy Physics 10, 041 (2007).

- J. Jose \&E. Saletan, Classical Dynamics: A Contemporary Approach, Cambridge University Press, (1998).

- M. De Laurentis, I. De Martino \& R. Lazkoz, Analysis of the Yukawa gravitational potential in $f(R)$ gravity II: relativistic periastron advance, Phys. Rev. (2018) D 97, 104068.

- J.D MeIss, Differential Dynamical Systems, SIAM, (2007).

- J.W Moffat, Scalar-Tensor-Vector Gravity Theory, J. Cosmol. Astropart. Phys., (2006) 3, 4, .

- J.W Moffat, A Modified Gravity and its Consequences for the Solar System, Astrophysics and Cosmology, International Journal of Modern Physics (2006) D16:20752090,2008

- H. Pollard, Mathematical Introduction to Celestial Mechanics, Prentice-Hall, Inc., (1966). 